This is the peer reviewed version of the following article: "Francesko, $\boldsymbol{A}$., Cano Fossas, M., Petkova, P., Fernandes, Margarida M., Mendoza, E., and Tzanov, Tzanko (2017) Sonochemical synthesis and stabilization of concentrated antimicrobial silver-chitosan nanoparticle dispersions. Journal of Applied Polymer Science, (134) 30: 45136 (1-8)." which has been published in final form at [doi: 10.1002lapp.45136]. This article may be used for non-commercial purposes in accordance with Wiley Terms and Conditions for Self-Archiving." 


\section{Sonochemical synthesis and stabilization of concentrated antimicrobial silver-chitosan nanoparticle dispersions}

Antonio Francesko ${ }^{\dagger}$, Marta Cano Fossas ${ }^{\dagger}$, Petya Petkova ${ }^{\dagger}$, Margarida M. Fernandes ${ }^{\dagger}$, Ernest Mendoza $^{\ddagger}$, Tzanko Tzanov ${ }^{\dagger} *$

$†$ Grup de Biotecnologia Molecular i Industrial, Department of Chemical Engineering, Universitat Politècnica de Catalunya, Spain ‡ Grup de Nanomaterials Aplicats. Centre de Recerca en Nanoenginyeria, Universitat Politècnica de Catalunya, Spain

* Corresponding author: Dr. Tzanko Tzanov

Grup de Biotecnologia Molecular i Industrial, Department of Chemical Engineering, Universitat Politècnica de Catalunya, Rambla Sant Nebridi 22, 08222, Terrassa, Spain

Tel: +34937398570; Fax: +34937398225

E-mail: tzanko.tzanov@upc.edu 


\begin{abstract}
This work reports on a green synthetic route to produce concentrated aqueous dispersions of silver nanoparticles (AgNP) employing high-intensity ultrasound (US) and chitosan (CS) as a non-toxic reducing agent for $\mathrm{Ag}^{+}$salts and AgNP stabilizer. The sonication simultaneously boosted the synthesis and improved the stability of the AgNP, capping them with CS. Hybrid AgNP-CS antimicrobial dispersions, stable for at least six months, were synthesized in a simple single step process. The use of US allowed for applying relatively mild processing temperatures $\left(60{ }^{\circ} \mathrm{C}\right)$ and reaction time between $30 \mathrm{~min}$ and $3 \mathrm{~h}$ to obtain concentrated dispersions of AgNP that otherwise could not be obtained even after $72 \mathrm{~h}$ under mechanical stirring at the same reaction conditions. Upon sonication spherical AgNP-CS with a size between 60 and $100 \mathrm{~nm}$ were generated, in contrast to the average diameter of $200 \mathrm{~nm}$ of the particles obtained by stirring. The antibacterial efficiency of the AgNP-CS hybrids was evaluated against the medically relevant pathogens Staphylococcus aureus and Escherichia coli. The US-synthesized AgNP-CS showed more than 3-fold higher antibacterial activity compared to the particles obtained under stirring, due to their higher concentration and smaller size.
\end{abstract}




\section{Introduction}

Silver nanoparticles (AgNP) are largely investigated antimicrobial agents applied in a wide range of consumer products, such as textiles, food packaging and cosmetics. Their long term impact, however, on both the human health and the environment is still largely unknown. Recent investigations suggest that there is still a need for technologically simple production of stable AgNP dispersions that are ecologically acceptable and biocompatible, responding to the safety issues related to their use and disposal. For example, the current materials loaded with AgNP are releasing particles during rinsing/washing that eventually go to the wastewater system and the environment, where they may become harmful to uncontrolled human exposure and also to microbes essential for the ecologic systems. ${ }^{1}$ AgNP were further reported to cross biological barriers in humans, e.g. blood-brain barrier and undesirably accumulate in different tissues. ${ }^{2}$

Biopolymers, such as polysaccharides, are therefore increasingly employed as capping agents for AgNP, anticipating multiple benefits from the formed nanohybrids. Polysaccharides would serve as: i) reducers in synthesis of AgNP from $\mathrm{Ag}^{+}$salts, being natural alternatives to the aggressive chemical reagents, ${ }^{3}$ ii) stabilizers of NP dispersions prolonging their shelf life, ${ }^{4}$ iii) protective agents that decrease the NP toxicity on human cells, ${ }^{5}$ iv) enhancers of the intrinsic antimicrobial properties of AgNP, ${ }^{6,7}$ and v) providers of the necessary functional groups for their permanent immobilization on solid substrates or their further surface functionalization. ${ }^{8}$

Chitosan (CS), in particular, is suitable for decorating AgNP, due to its positive charge that avoids the particles aggregation. At the same time, the formed CS shell around the AgNP improves compatibility of AgNP with human cells and tissues. Capping of AgNP with CS can also result in higher bactericidal effect on planktonic bacteria compared to CS or AgNP alone, 
most notably due to the cationic character of CS that causes disturbance of the bacterial membranes. $^{9-12}$

Another important benefit of using CS is the possibility to exploit the green synthesis of AgNP, replacing harsh chemical reagents, such as $\mathrm{NaBH}_{4}$. In a green synthetic approach, CS reduces $\mathrm{Ag}^{+}$to elemental silver, while its macromolecules simultaneously interact with the surface of the formed AgNP and stabilize them. ${ }^{13} \mathrm{Ag}^{+}$reduction, however, occurs only if the mixture is heated above $70{ }^{\circ} \mathrm{C}$. Maintaining elevated temperature during several days' reaction can be detrimental for the natural polymers, besides of being industrially unattractive. Thus, the unmet challenge for the large-scale green synthesis of AgNP is to simultaneously increase the reaction yields while decreasing the temperature and duration of the process.

To address this challenge, different technologies have been suggested to facilitate the $\mathrm{Ag}^{+}$ reduction in presence of biopolymers. These synthesis intensification technologies are typically based on increasing the local temperature and/or pressure using UV-irradiation, ${ }^{14}$ microwave, ${ }^{15,16}$ and autoclave. ${ }^{17}$ However, the size of the obtained hybrids is insufficiently controllable with temperature, reaction time and ratio of reagents used, in addition to the low concentration of the AgNP dispersion, compromising the industrial feasibility of the current green approaches.

Sonochemistry is another, recently highly exploited technology to considerably enhance the reduction of $\mathrm{Ag}^{+}$in presence of different stabilizers. ${ }^{18-20}$ This approach employs a powerful ultrasound (US) radiation ( $\geq 20 \mathrm{kHz}$ ) to chemically transform molecules in the so called "hot spot mechanism”. The physical phenomenon associated with US is the acoustic cavitation - the formation, growth, and implosive collapse of bubbles in a liquid. The energy released during the bubbles collapse permits a range of chemical reactions and the synthesis of a wide variety of nanostructured materials. ${ }^{21}$ From industrial point of view, the sonochemistry is an extremely 
versatile one-step tool for emulsification and nanofabrication. The latter includes the preparation of amorphous nanoarchitectures, insertion of nanoobjects into mesoporous materials, deposition of nanoparticles on functional surfaces and formation of soft nano/micro-spheres. ${ }^{22}$ In our previous studies, the sonochemistry was employed to transform polysaccharides, proteins, phenolic compounds and synthetic antibiotics into bioactive NP for a broad range of medical and cosmetic applications. ${ }^{23-28}$

The current study attempts to achieve rapid green synthesis of stable and concentrated dispersions of CS-capped AgNP in a one-step sonochemical process for CS-assisted reduction of $\mathrm{Ag}^{+}$into AgNP. To the best of our knowledge, this is the first trial to obtain highly concentrated AgNP-CS dispersions at reduced synthesis time using sonochemistry as an alternative to the mechanical stirring. The study further contrasts the reaction yields and NP size/distribution of the sonochemically-processed AgNP-CS hybrids and their counterparts obtained by stirring. Since little is known on how the different manufacturing technologies influence the antimicrobial capacity of the AgNP, we applied the hybrids for eradication of medically relevant Grampositive and Gram-negative strains.

\section{Experimental section}

\subsection{Materials and reagents}

Medical grade CS ( $\sim 15 \mathrm{kDa}$, DDA $87 \%)$ was purchased from Kitozyme (Belgium). Silver nitrate $\left(\mathrm{AgNO}_{3}\right)$, (3-glycidyloxypropyl)trimethoxysilane (GOPTES), 4-(4-nitrobenzyl)pyridine (NBP), acetic acid, hydrochloric acid, sodium hydroxide and ethanol were of analytical grade 
purchased from Sigma-Aldrich (Spain). Gram-positive Staphylococcus aureus ATCC 25923 (S. aureus) and Gram-negative Escherichia coli ATCC 25922 (E. coli) bacteria were used for the antimicrobial assays. Baird-Parker agar and Coliform agar for bacteria culturing and other reagents for antimicrobial studies were purchased from Sigma-Aldrich unless otherwise specified.

\subsection{Methods}

\subsubsection{Synthesis of AgNP-CS}

For the AgNP-CS preparation, $20 \mathrm{~mL}$ of $\mathrm{AgNO}_{3}(2 \mathrm{mg} / \mathrm{mL})$ were mixed with $30 \mathrm{~mL}$ of $\mathrm{CS}$ solution ( $1 \% \mathrm{w} / \mathrm{v}$ prepared in $1 \%$ acetic acid, $\mathrm{pH}$ adjusted to 5.5 with $3 \mathrm{M}$ sodium hydroxide). The mixture was then subjected to US irradiation for up to $3 \mathrm{~h}$ with an ultrasonic transducer Tihorn $20 \mathrm{kHz}$ (Sonics and Materials VC750, USA) equipped with thermostatic bath to maintain the reaction temperature at $60{ }^{\circ} \mathrm{C}$. By measuring the time-dependent increase of the temperature in the ultrasonic glass jacketed vessel, the US intensity $\left(17.30 \mathrm{~W} / \mathrm{cm}^{2}\right)$, density $\left(0.43 \mathrm{~W} / \mathrm{cm}^{3}\right)$ and power $(21.5 \mathrm{~W})$ used for the synthesis were determined calorimetrically. In contrast, the control suspension was stirred at the same temperature for up to $72 \mathrm{~h}$. At several different time points $200 \mu \mathrm{L}$ of each suspension were removed for UV-vis analysis.

\subsubsection{Characterization of AgNP-CS}

Spectrophotometric measurements were performed in a microplate reader Infinite M200, Tecan (Austria). The UV-vis spectra were collected in the range of $300-600 \mathrm{~nm}$, recording absorbance at a $2 \mathrm{~nm}$ step. 
Nanoparticle tracking analysis (NTA) was used to characterize the AgNP-CS dispersions. The measurements were performed in a NTA LM10HSBF instrument (Nanosight, UK) equipped with a $60 \mathrm{~mW}$ at $450 \mathrm{~nm}$ laser, a high-resolution Complementary Metal Oxide Silicon (CMOS) camera, and a thermal detector. NTA Software v2.3 was used to capture several frames of the NPs dispersions and correlate the particle size with the sample concentration. Prior to measurements the software sensitivity was optimized to detect concentrations greater than $10^{7}$ particles per mL. Despite the sensitivity adjustments, the AgNP-CS samples (stirred and sonicated) had to be diluted 1000 times. The Brownian motion in the NPs dispersions was recorded for ca. 10 min at room temperature, and the data were subsequently analyzed with the software that simultaneously tracks and sizes the visualized particles. Results are displayed as distribution plots of concentration (particles/mL) vs. particle size. The reported average particle sizes represent the mean values \pm standard deviations of three independent measurements per sample.

The AgNPs-CS were further visualized by a Zeiss Neon FIB microscope (Carl Zeiss, Germany) operating in scanning transmission electron microscopy (STEM) mode at $30 \mathrm{kV}$ acceleration voltage. The NP dispersions were previously diluted 100 times, and $20 \mu \mathrm{L}$ of the diluted samples were drop-casted on a TEM holey carbon grid.

Fourier transform infrared spectroscopy (FTIR) was used to analyze the presence of CS in the NP suspension. A Perkin Elmer Spectrum 100 (USA) equipped with ATR sampling accessory was used to record the IR spectra between $4000-600 \mathrm{~cm}^{-1}$, performing 64 scans for each spectrum. Silicone strips, more specifically polydimethyl/vinilmethyl siloxane (PDMS) (supplied by Degania Silicone Ltd., Israel) were used as surfaces for deposition of the hybrid AgNP-CS from the dispersion. The strips were previously activated with an epoxy reagent (GOPTES) in 
order to provide epoxy groups for CS grafting (via amines) on their surface. Similar epoxyamino conjugation reaction was recently exploited in grafting proteins onto silicon-based transducers of various biosensing devices. ${ }^{29}$ Briefly, the stripes were plasma-activated at 13.56 $\mathrm{MHz}$ and $100 \mathrm{~W}$ for $5 \mathrm{~min}$, in a radiofrequency reactor with oxygen as plasma gas supplied at a constant flow rate of $15 \mathrm{~cm}^{3} / \mathrm{min}$ to generate predominantly $\mathrm{OH}^{-}$on the silicone (PDMS) surface. The samples were immediately transferred to $5 \%$ (v/v) GOPTES in ethanol and left for $24 \mathrm{~h}$ in a dark place and RT. The unbound GOPTES was removed by washing with ethanol. The availability of epoxy groups on the surface was qualitatively confirmed by immersing the samples in $40 \mathrm{mg} / \mathrm{mL}$ NBP in 2-methoxyethanol for $2 \mathrm{~h}$ at $80{ }^{\circ} \mathrm{C}$, obtaining a characteristic purple color. GOPTES treated PDMS strips were then immersed in the AgNP-CS sonicated suspension and incubated in a thermostated laboratory shaker at $50{ }^{\circ} \mathrm{C}$ and $80 \mathrm{rpm}$ overnight. The loosely bound NPs were removed by thorough washing with distilled water after which the samples were dried under $\mathrm{N}_{2}$. Control sample was prepared in the same way without prefunctionalization of the silicone strip.

Scanning electron microscopy (SEM) obtained using a Zeiss Neon FIB microscope (Carl Zeiss, Germany) was performed to visualize the AgNPs-CS hybrids casted or chemically immobilized on the silicone PDMS surface.

\subsubsection{Antimicrobial activity}

The antibacterial effect of the AgNP-CS dispersions (prepared under stirring or US) was assessed against the Gram-positive S. aureus and the Gram-negative E. coli. A single colony from the corresponding bacterial cultures was used for the preparation of the bacterial suspensions. The culture was then incubated at $37^{\circ} \mathrm{C}$ and $230 \mathrm{rpm}$ and grown overnight in sterile 
nutrient broth (NB, Sharlab, Spain). In order to obtain the bacteria stock solutions, the overnight grown bacteria culture was diluted with NB until reaching the absorbance of 0.01 at $600 \mathrm{~nm}$. Thereafter, $250 \mu \mathrm{L}$ of AgNPs-CS dispersions (diluted in NB 50, 100, and 150 times) were incubated with $250 \mu \mathrm{L}$ of bacteria stock solution for $24 \mathrm{~h}$ at $37^{\circ} \mathrm{C}$ and $230 \mathrm{rpm}$. Thereafter, different dilutions of the suspensions (prepared in sterile $0.3 \mathrm{mM} \mathrm{KH}_{2} \mathrm{PO}_{4}$ ) were plated on a Baird-Parker and Coliform agar (for S. aureus and E. coli, respectively) and grown at $37^{\circ} \mathrm{C}$ for $24 \mathrm{~h}$. The number of viable bacterial colonies were finally counted and recalculated to CFU/mL of suspension. The results reported are the mean values \pm standard deviation of three independent measurements per AgNP-CS dispersion.

\section{Results and discussion}

\subsection{Synthesis of AgNPs-CS}

The AgNP formation was followed by UV-vis spectrophotometry showing absorbance spectra with typical peak for silver ${ }^{30}$ at around $420 \mathrm{~nm}$ (Fig. 1). Over time, the peak intensity considerably differed between the mechanically stirred and sonicated NP dispersions. Whereas for the stirred dispersions a small shoulder appeared only after $24 \mathrm{~h}$, the US irradiation brought about NP formation already after 30 min, as denoted by the appearance of the peak at the same wavelength (420 nm). Its intensity noticeably increased during $3 \mathrm{~h}$. In addition, the differences in formation of colloidal NP suspensions upon stirring and sonication were visible also on the photographs of the AgNP-CS dispersions. A light yellow color appeared in the stirred dispersion

during the first $3 \mathrm{~h}$, while the color of the US-assisted AgNP-CS system was comparatively stronger for the same process duration, suggesting an enhanced NP synthesis. The typical 
yellowish color and the absorbance peak at $420 \mathrm{~nm}$ proceed from the excitation of surface plasmon vibrations of Ag atoms. ${ }^{31}$

Although $\mathrm{Ag}^{+}$reduction occurred both under stirring and sonication, the AgNP synthesis was greatly accelerated by the high-power US irradiation. In comparison, the widely reported green synthesis of metal NP yields reasonably concentrated dispersions only in a several days' process, as confirmed in our stirring experiment. Creating high local temperatures in the mixture, the sonication boosted the synthesis and stabilization of AgNP-CS. ${ }^{32}$ From mechanistic point of view, the acoustic cavitation in liquids caused by US produces high local temperatures and pressures leading to rapid decomposition of the metal precursors to metal atoms agglomerating into NP, provided that an appropriate stabilizing agent, such as CS is present to avoid their precipitation. ${ }^{21,33}$ That is why in order to achieve the same NP yield by sonication, the quantity of precursor and reducer/stabilizer are comparatively lower than in the case of mechanical stirring. However, unlike in most of the synthetic procedures found in literature, the goal of this study was to compare the exploitation characteristics of the AgNP dispersions obtained upon stirring and US starting from the same reaction composition. It is worth mentioning that no aggregates were observed for more than 6 months after preparation, even in the concentrated US dispersion, which indicates the high stability of the NP regardless of the synthesis approach - stirring or US. 

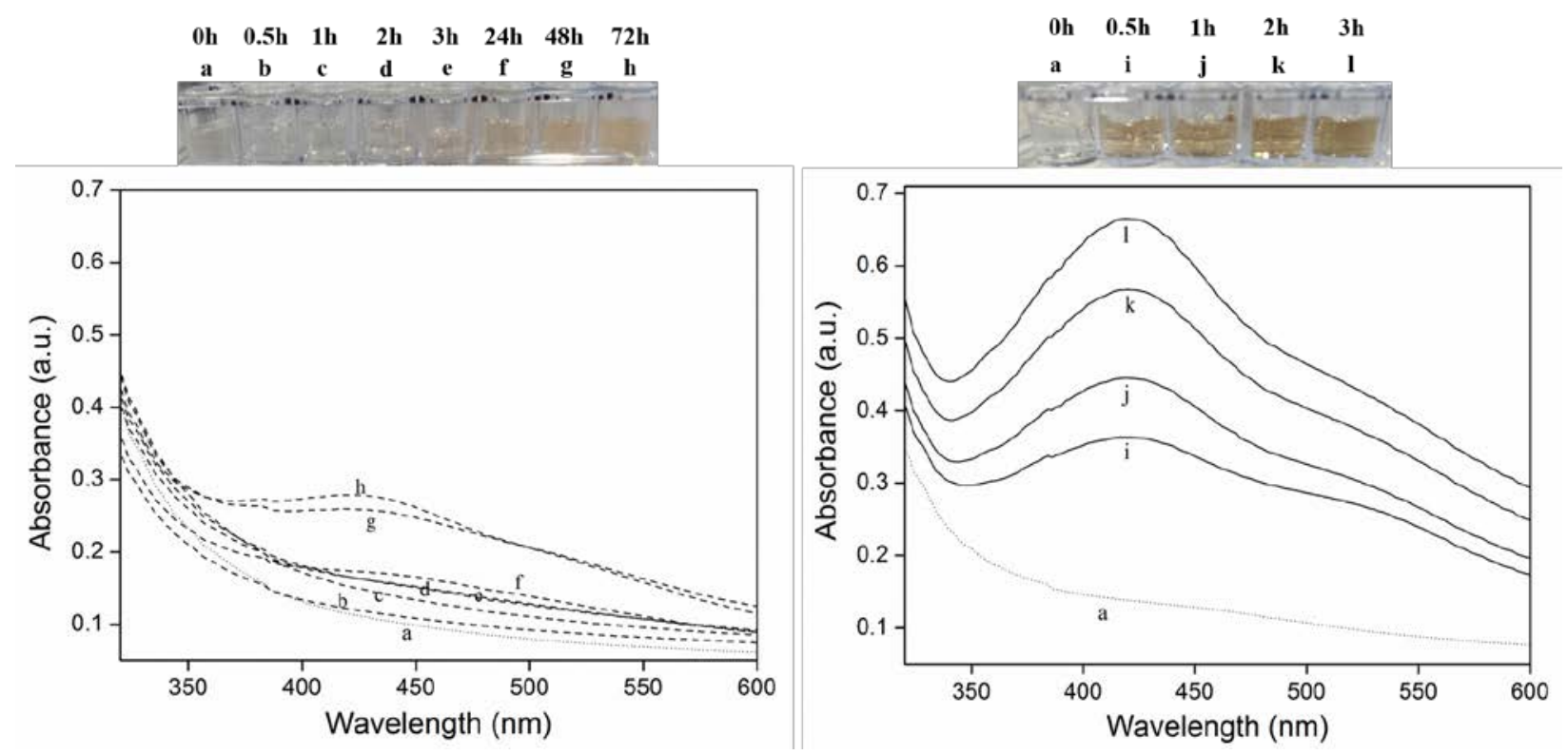

Figure 1. UV-vis spectra and photographs of control (a, AgNP-CS mixture at $0 \mathrm{~h}$ ) and AgNP-CS dispersions varying the stirring (left graph, b-h) and sonication (right graph, i-l).

\subsection{Characterization of AgNPs-CS}

NTA technique is a simple, rapid and reliable characterization method that allows precise measurement of the size distribution of colloidal suspensions. The instrument records the Brownian motion of the colloidal nanoentities and is able to average the distances travelled by the particles and relate their diffusion with size. The technique has been used to compare capping effect of a variety of biocompounds to stabilize colloidal metal NPs, including Ag and Au, and finding a good agreement with the results obtained by TEM, and thus setting this technique as a reliable tool for the assessment of NP size and distribution. ${ }^{34,35}$ In this study NTA was employed to qualitatively and quantitatively compare the AgNP-CS hybrids obtained by means of mechanical stirring and sonication. 
Differences in the size and distribution patterns were observed between the stirred and the sonicated AgNP-CS dispersions. As suggested by UV-Vis measurements, the sonication of the $\mathrm{Ag}^{+} / \mathrm{CS}$ mixture brought about the formation of a greater number of smaller NP even during the significantly shorter synthesis time $(3 \mathrm{~h})$, in contrast to the $72 \mathrm{~h}$ synthesis under stirring (Table 1). The average AgNP-CS size was around $200 \mathrm{~nm}$ for the stirred dispersion and $100 \mathrm{~nm}$ for the sonicated one. The sonicated dispersion contained roughly 1.5 times more particles than the stirred one, clearly promoting the simultaneous $\mathrm{Ag}^{+}$reduction and particles capping with CS. Thus, the use of US is determinant for more efficient AgNP capping with CS and thereby surface energy reduction of the colloidal silver, providing increased stability of the dispersion. Similarly, we have previously obtained stable polysaccharide nanospheres without the use of any surfactant with the assistance of US. ${ }^{23}$

Table 1. Comparative particle size and concentration measured by NTA for stable AgNP-CS colloidal dispersions.

\begin{tabular}{|c|c|c|}
\hline AgNP-CS dispersion & Particle size (nm) & Particle concentration $\left(\mathbf{x 1 0}^{-11}\right.$ particles/mL) \\
\hline Stirred for 72 h & $197.7 \pm 2.3$ & $4.72 \pm 0.54$ \\
\hline Sonicated for 3 h & $94.7 \pm 3.6$ & $6.70 \pm 0.44$ \\
\hline
\end{tabular}

In addition to the average size determination and quantification of the particles, NTA also allowed evaluating their distribution, discriminating between particles size subtypes (Fig. 2). The distribution profile obtained by NTA showed that the particles obtained during $72 \mathrm{~h}$ mechanical stirring display a wide distribution profile with three large populations of each size subtype at 95, 151 and $224 \mathrm{~nm}$ (Fig. 2, left graph). In contrast, the US-assisted dispersion in only $3 \mathrm{~h}$ displayed 
predominantly two size subtypes at $62 \mathrm{~nm}$ and $100 \mathrm{~nm}$, with narrower distribution profile and considerably lower polydispersity (Fig. 2, right graph). It is worth mentioning that in the sonicated dispersion the $62 \mathrm{~nm}$ subtype size contained nearly 3 times more NP than the $100 \mathrm{~nm}$ subtype size. Significantly lower populations represented the other size subtypes in the stirred and the sonicated dispersions. Thus, besides improving the NP reaction yield, the US also promoted the synthesis of smaller particles with lower polydispersity index compared to mechanical stirring.
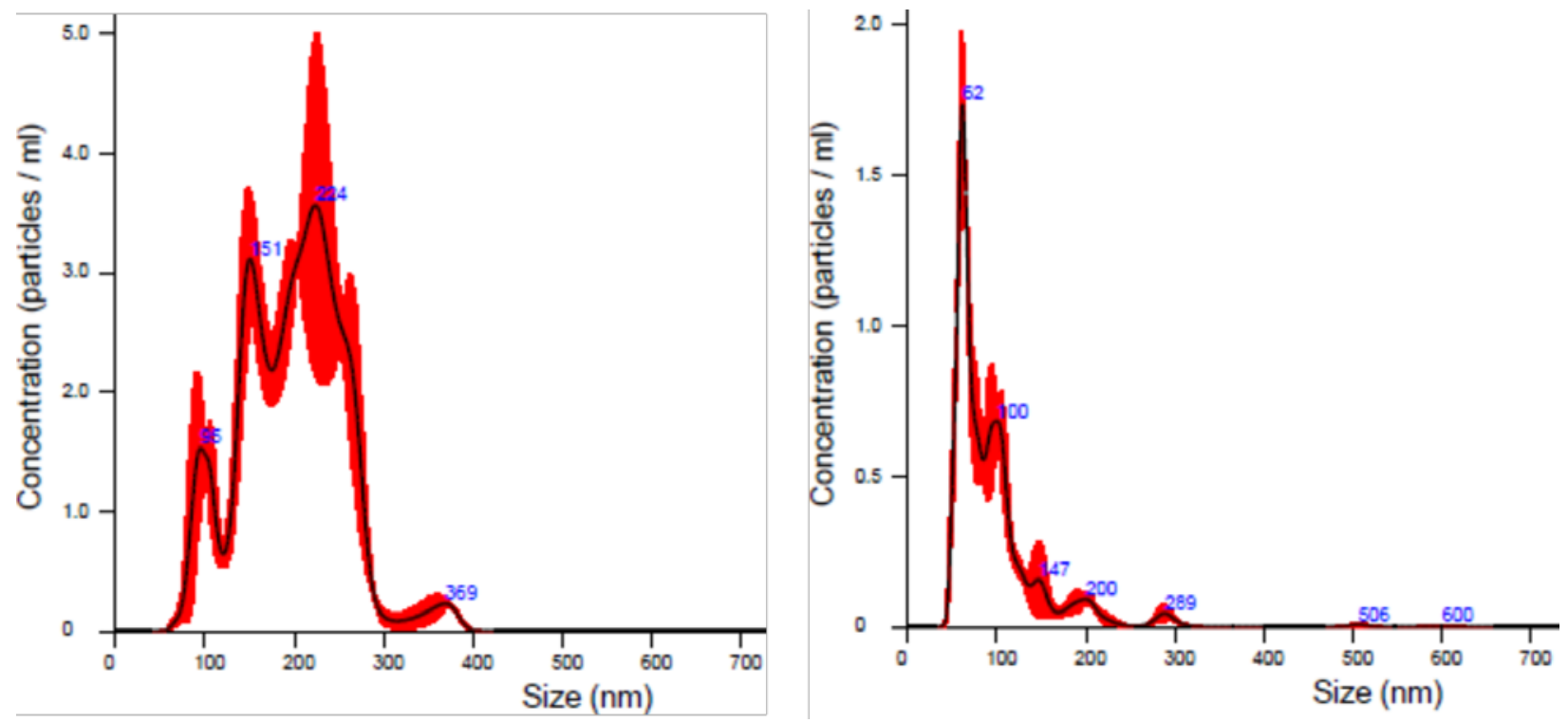

Figure 2. Concentration vs. size plots of AgNP-CS dispersions synthesized during $72 \mathrm{~h}$ stirring (left graph) and 3 h sonication (right graph). Only qualitative concentration comparison should be made between the samples. ${ }^{34}$ 
Based on the UV-vis and NTA analyses the overview of the technological approaches and the resulting hybrid dispersions is represented on Scheme 1. The images were taken to note the difference in the synthesis time between sonication (3 h) and stirring (72 h).

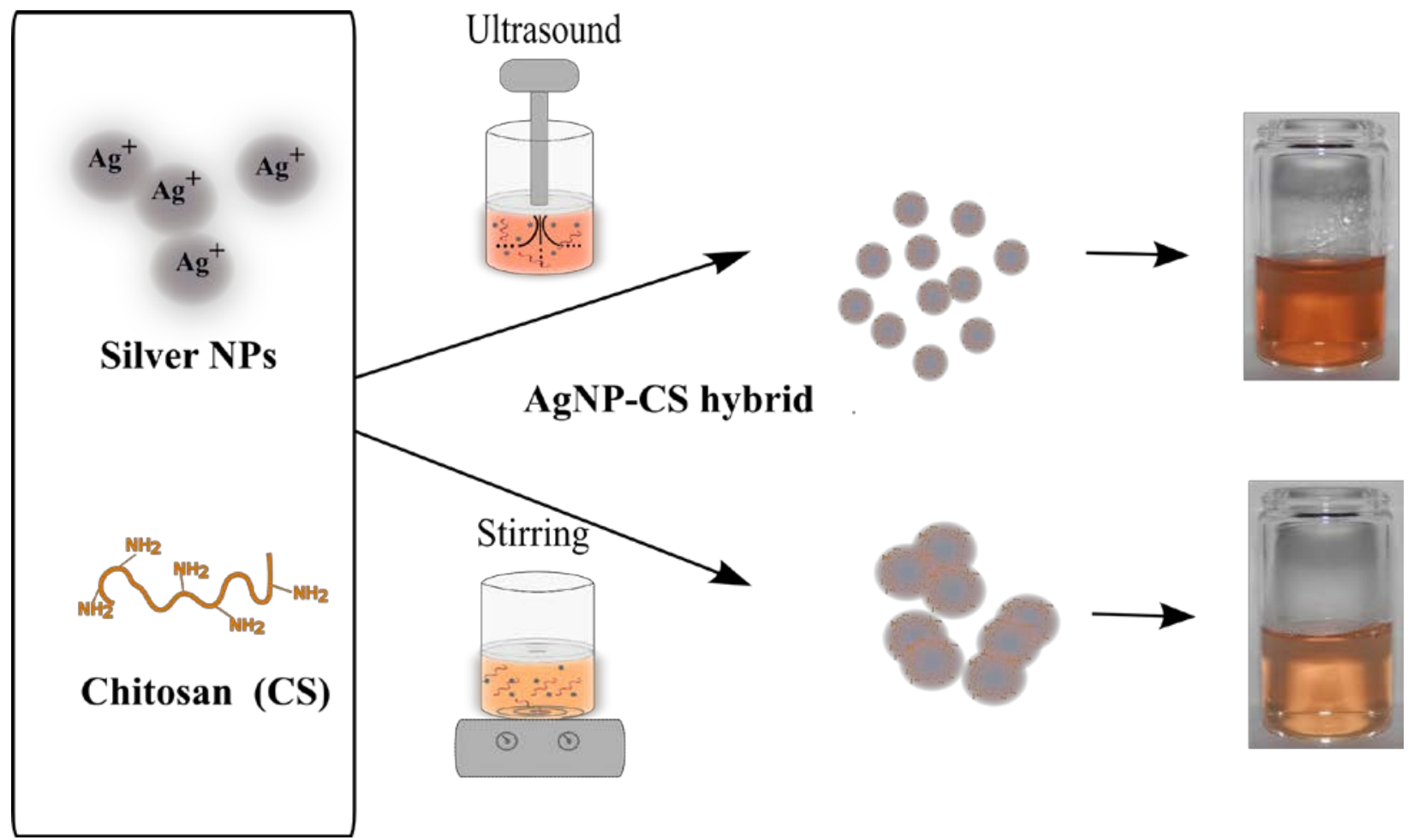

Scheme 1. Synthesis of AgNP-CS hybrids via US and stirring. The images of the resulting dispersions are on the right side and are taken after $3 \mathrm{~h}$ of synthesis using sonication and $72 \mathrm{~h}$ of synthesis using mechanical stirring.

STEM analysis confirmed the larger number and smaller size of the AgNP-CS obtained in the US-assisted process (Fig. 3, inset images show photographs of the $72 \mathrm{~h}$ stirred and $3 \mathrm{~h}$ sonicated dispersion). The AgNPs-CS from both the stirred and sonicated dispersion were shaped in sphere-like structures, which for the US-assisted dispersion did not exceed $100 \mathrm{~nm}$, thereby 
corroborating the NTA findings. In contrast, NP complexes were identified in the stirred dispersion, which could be related to lower NP stability and aggregation during drying the sample on the TEM grid. The larger number of smaller NP in the sonicated dispersion also suggests that the US waves, besides of boosting the NP synthesis and stabilization, further disperse the nanomaterials in the suspension.
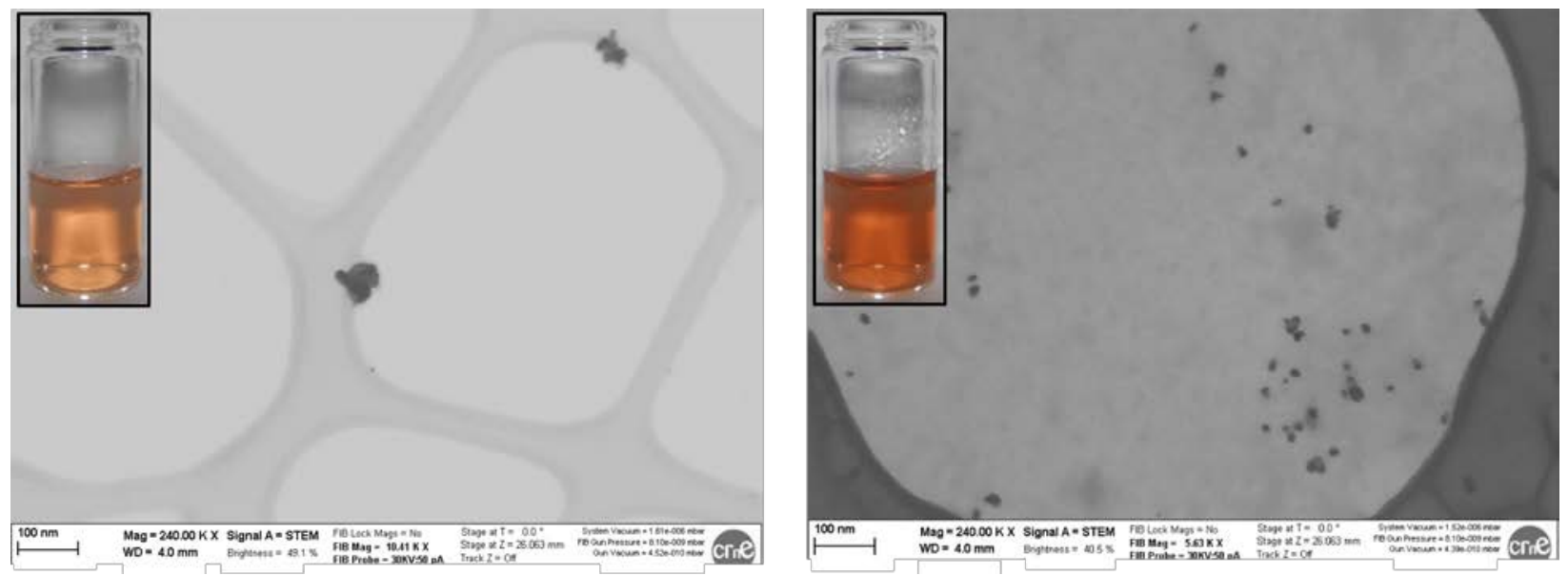

Figure 3. STEM images of the AgNPs-CS hybrids synthesized during $72 \mathrm{~h}$ stirring (left image) and $3 \mathrm{~h}$ sonication (right image). The appearance of the analyzed dispersions is shown in the inset photographs.

FTIR analysis was used to confirm the capping of AgNP with CS. To this end, the AgNP-CS hybrids were grafted on a silicone (PDMS) surface pre-activated with epoxy-silane (GOPTES). Amine containing macromolecules, such as CS in our AgNP-CS hybrids, can be covalently bound to epoxy-modified silicones, and remain on their surface even after thoroughly washing the material. This was the case in our previous work with core-shell nano-/micro-spheres grafted 
on silicone PDMS via epoxy-amino chemistry. ${ }^{36}$ SEM images were further taken to confirm the permanent functionalization of silicone with both stirred and sonicated AgNP-CS hybrids. Importantly, the SEM analysis did not detect the presence of AgNP-CS hybrids on the control pristine silicone surface (result not shown). Consequently, the FTIR spectrum did not display any of the characteristic peaks of CS (Fig. 4, dotted line). In contrast, in the spectra of the epoxy preactivated silicone, one of the most prominent CS bands in the polysaccharide region between 1500 and $1700 \mathrm{~cm}^{-1}$ associated with the presence of CS amines, confirmed the successful grafting of AgNP-CS, regardless of their method of synthesis, i.e. stirring or sonication (Fig. 4, dashed and solid lines). The intensive signals in the region between 3000 and $3500 \mathrm{~cm}^{-1}$ are also typical for the stretching vibrations of $\mathrm{N}-\mathrm{H}$ and $\mathrm{O}-\mathrm{H}$ in CS. It is worth mentioning that after normalization of the spectra, these typical for CS signals were more intensive for sonicated AgNP-CS compared to the stirred hybrid. It is reasonable to expect that a more concentrated sonicated dispersion will result in a larger number of NP hybrids bound to the activated silicone surface. However, this could not be verified with SEM analysis, since the presence of a considerable number of homogeneously distributed NP on the silicone were found for both stirred and sonicated hybrids (Fig. 4, SEM images). On the other hand, SEM images further corroborated the FTIR findings that CS decorated AgNP are covalently conjugated onto the epoxy pre-activated silicone, since thorough washings did not result in their removal. 

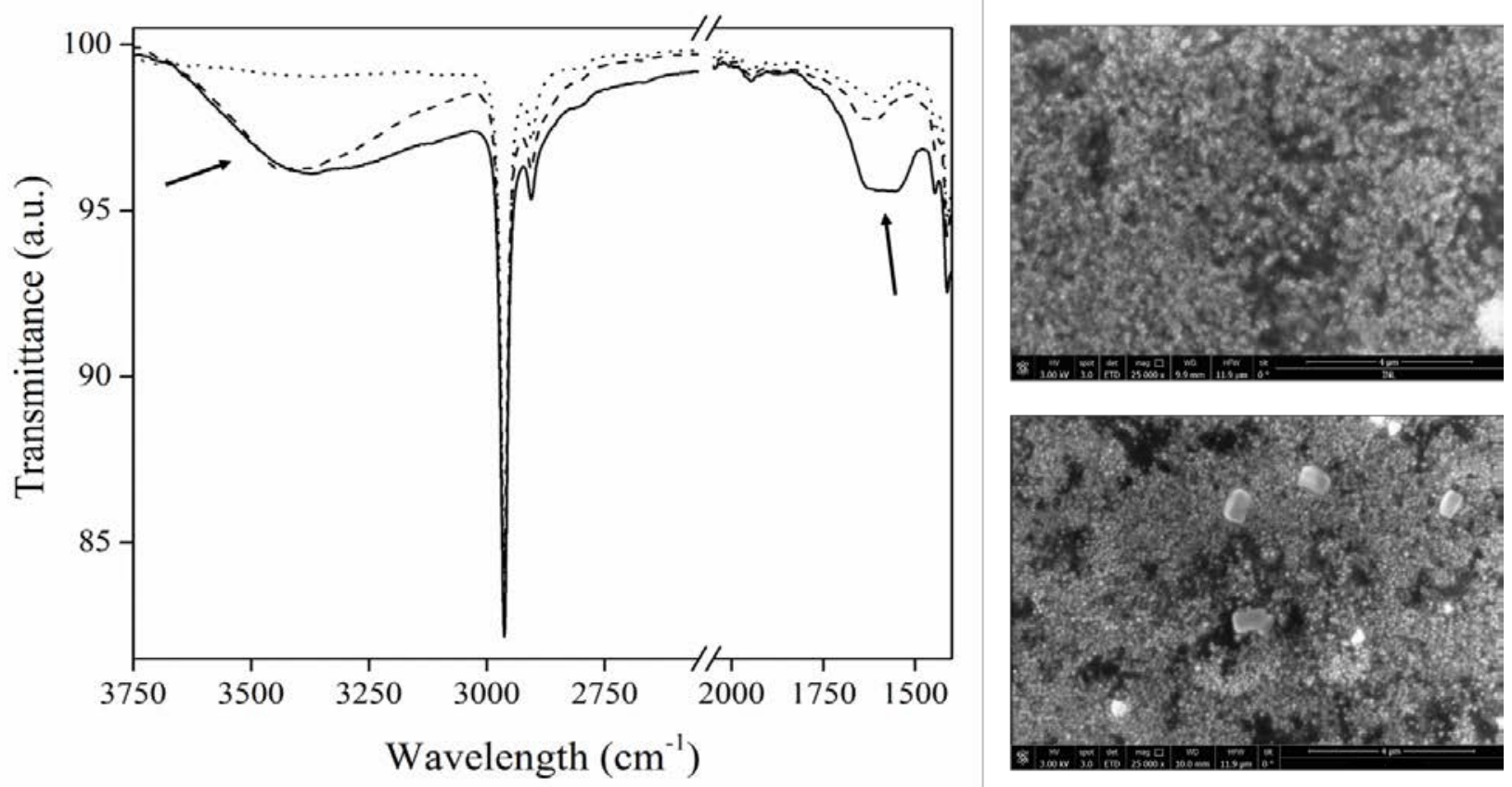

Figure 4. Normalized FTIR spectra of control silicone PDMS (dotted line) and epoxy-activated silicone PDMS surfaces treated with $72 \mathrm{~h}$ stirred (dashed line) and $3 \mathrm{~h}$ sonicated (solid line) dispersion of AgNP-CS. The treated surfaces were subsequently subjected to thorough washings to confirm the permanent binding. The SEM images $(\times 25 \mathrm{~K})$ with scale bars of $4 \mu \mathrm{m}$ represent the analyzed epoxy-silicone treated with stirred (top image) and sonicated (bottom image) AgNP-CS dispersions.

\subsection{Antibacterial efficiency of AgNPs-CS}

The antibacterial properties of the hybrid AgNP-CS were assessed against planktonic S. aureus and E. coli, which are among the most clinically relevant bacteria responsible for the current global medical challenge of hospital-acquired infections. For example, pathogenic Gramnegative E. coli strains easily colonize the surface of Foley catheters causing complicated urinary tract infections, ${ }^{37}$ whereas Gram-positive S. aureus strains usually originate from the skin of 
patients or health-care workers. ${ }^{38}$ Silver has been recognized as an efficient and broad range antibacterial material, especially when its size is brought down to the nanometer range due to the increased surface to volume ratio. ${ }^{39}$ Moreover, the inclusion of a biopolymer such as CS, able to interfere and disrupt the bacterial membrane, ${ }^{23}$ as a capping and stabilizing agent is thought to synergistically contribute to the bactericidal and antibiofilm mechanisms of the metalbiopolymer hybrids. ${ }^{6}$

In order to assess the antimicrobial properties of the AgNPs-CS dispersions and find their optimal range of effectiveness, the dispersions were diluted 50, 100, and 150 times in NB, and their ability to eradicate the above-mentioned microorganisms was assessed. The less concentrated samples (x150 diluted) did not show antimicrobial activity (Fig. 5). However, when a more concentrated sample was analyzed (x100 diluted), a bactericidal effect was observed. This effect was more pronounced when the particles were obtained using US, inducing $72 \%$ and $65 \%$ inhibition of S. aureus and E. coli respectively, while the particles obtained by stirring only inhibited $20 \%$ of S. aureus and $16 \%$ E. coli. The differences in size and quantity of the particles obtained by the two methods, e.g. $\sim 100 \mathrm{~nm}$ for US-generated particles and $\sim 200 \mathrm{~nm}$ for the stirred dispersion, are certainly the reason for their different antimicrobial capacities. Finally, when the dispersions were diluted x50, a full kill effect was observed for the AgNP-CS produced by both US and stirring techniques. 

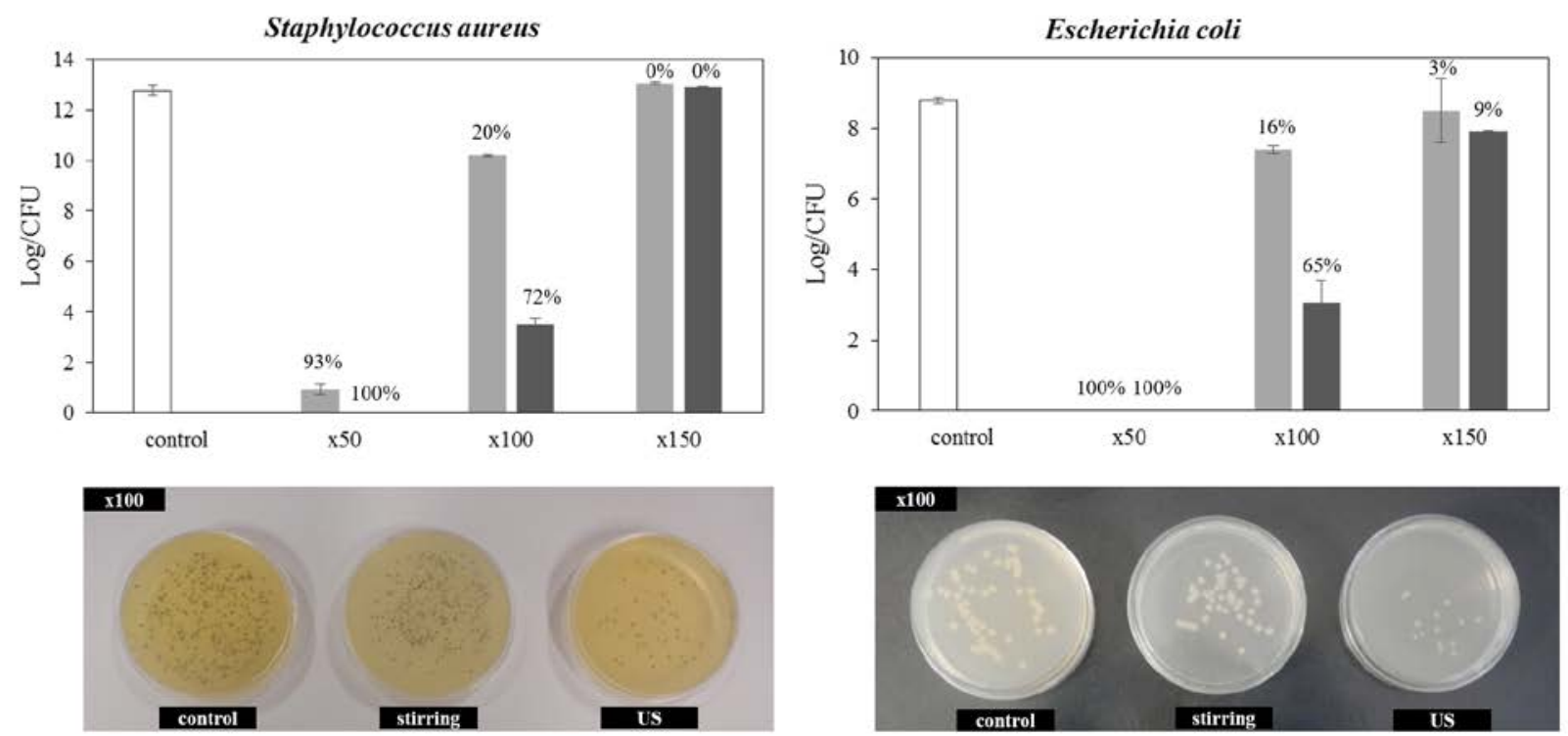

Figure 5. Antibacterial activity of AgNPs-CS dispersions diluted 50, 100 and 150 times in NB.

The dispersions were obtained after $72 \mathrm{~h}$ stirring (light grey bars) and $3 \mathrm{~h}$ in the US-assisted process (dark grey bars). The respective photographs of CFU in the plates were given below the graphs analyzed for x100 diluted dispersions.

\section{Conclusions}

In summary, an environmentally friendly single-step sonochemical route was developed and optimized for the fabrication of stable and concentrated antimicrobial dispersions of CS-capped AgNP. The feasibility of the US-assisted NP synthesis was demonstrated under industrially attractive process conditions, e.g. short, up to $3 \mathrm{~h}$ reaction time and mild process temperatures $\left(60^{\circ} \mathrm{C}\right)$. On the other hand, CS played a dual role in the reaction: i) effectively reducing $\mathrm{Ag}^{+}$to elemental silver, and ii) stabilizing by capping the generated AgNP. Finally, antimicrobial studies showed that the AgNP-CS hybrids efficiently inhibited the growth of E. coli and $S$. 
aureus. The easy manufacturing of highly antibacterial NP dispersions by US is potentially exploitable for preparation of hybrid biomedical materials for efficient infection control and bacterial biofilm inhibition.

\section{References}

1. Voelker, D., Schlich, K., Hohndorf, L., Koch, W., Kuehnen, U., Polleichtner, C., Kussatz, C. and Hund-Rinke, K. Environ. Res. 2015, 140, 661-672.

2. Trickler, W. J., Lantz, S. M., Murdock, R. C., Schrand, A. M., Robinson, B. L., Newport, G. D., Schlager, J. J., Oldenburg, S. J., Paule, M. G., Slikker, W., Hussain, S. M. and Ali, S. F. Toxicol. Sci. 2010, 118, 160-170.

3. $\quad$ Park, Y., Hong, Y. N., Weyers, A., Kim, Y. S. and Linhardt, R. J. IET Nanobiotechnol. 2011, 5, 69-78.

4. Srikar, S. K., Giri, D. D., Pal, D. B., Mishra, P. K. and Upadhyay, S. N. Green Sustainable Chem. 2016, 6, 34-56.

5. Petkova, P., Francesko, A., Fernandes, M. M., Mendoza, E., Perelshtein, I., Gedanken, A. and Tzanov, T. ACS Appl. Mater. Interface 2014, 6, 1164-1172.

6. Sanyasi, S., Majhi, R. K., Kumar, S., Mishra, M., Ghosh, A., Suar, M., Satyam, P. V., Mohapatra, H., Goswami, C. and Goswami, L. Sci. Reports 2016, 6, 24929-24945.

7. Potara, M., Jakab, E., Damert, A., Popescu, O., Canpean, V. and Astilean, S. Nanotechnology 2011, 22, 135101-135110.

8. Francesko, A., Blandón, L., Vázquez, M., Petkova, P., Morató, J., Pfeifer, A., Heinze, T., Mendoza, E. and Tzanov, T. ACS Appl. Mater. Interface 2015, 7, 9792-9799.

9. Wei, D., Sun, W., Qian, W., Ye, Y. and Ma, X. Carbohyd. Res. 2009, 344, 2375-2382. 
10. An, J., Luo, Q., Yuan, X., Wang, D. and Li, X. J. Appl. Polym. Sci. 2011, 120, 31803189.

11. Sanpui, P., Murugadoss, A., Prasad, P. V. D., Ghosh, S. S. and Chattopadhyay, A. Int. J. Food Microbiol. 2008, 124, 142-146.

12. Wang, L.-S., Wang, C.-Y., Yang, C.-H., Hsieh, C.-L., Chen, S.-Y., Shen, C.-Y., Wang, J.-J. and Huang, K.-S. Int. J. Nanomed. 2015, 10, 2685-2696.

13. Twu, Y.-K., Chen, Y.-W. and Shih, C.-M. Powder Technol. 2008, 185, 251-257.

14. Huang, L., Zhai, M. L., Long, D. W., Peng, J., Xu, L., Wu, G. Z., Li, J. Q. and Wei, G. S. J. Nanopart. Res. 2008, 10, 1193-1202.

15. Nguyen, N. T., Nguyen, B. H., Ba, D. T., Pham, D. G., Van Khai, T., Nguyen, L. T. and Tran, L. D. Mater. Manuf. Process. 2014, 29, 418-421.

16. Kahrilas, G. A., Wally, L. M., Fredrick, S. J., Hiskey, M., Prieto, A. L. and Owens, J. E. ACS Sustainable Chem. Eng. 2014, 2, 367-376.

17. Venkatesham, M., Ayodhya, D., Madhusudhan, A., Veera Babu, N. and Veerabhadram, G. Appl. Nanosci. 2014, 4, 113-119.

18. Wani, I. A., Ganguly, A., Ahmed, J. and Ahmad, T. Mater. Lett. 2011, 65, 520-522.

19. Gautam, S., Dubey, P. and Gupta, M. N. Colloid Surface B 2013, 102, 879-883.

20. Kumar, B., Smita, K., Cumbal, L., Debut, A. and Pathak, R. N. Bioinorg. Chem. Appl. 2014, 2014, Article ID 784268.

21. Bang, J. H. and Suslick, K. S. Adv. Mater. 2010, 22, 1039-1059.

22. Gedanken, A. Ultrason. Sonochem. 2004, 11, 47-55.

23. Fernandes, M. M., Francesko, A., Torrent-Burgués, J., Carrión-Fité, F. J., Heinze, T. and Tzanov, T. Biomacromolecules 2014, 15, 1365-1374. 
24. Francesko, A., Fernandes, M. M., Perelshtein, I., Benisvy-Aharonovich, E., Gedanken, A. and Tzanov, T. J. Mater. Chem. B 2014, 2, 6020-6029.

25. Fernandes, M. M., Rivera, D., Francesko, A., Šližytė, R., Mozuraitytė, R., Rommi, K., Lantto, R. and Tzanov, T. Process Biochem. 2015, 50, 1843-1851.

26. Fernandes, M. M., Ivanova, K., Francesko, A., Rivera, D., Torrent-Burgués, J., Gedanken, A., Mendonza, E. and Tzanov, T. Nanomed-Nanotechnol. 2016, 12, 2061-2069.

27. Petkova, P., Francesko, A., Perelshtein, I., Gedanken, A. and Tzanov, T. Ultrason. Sonochem. 2016, 29, 244-250.

28. Francesko, A., Fernandes, M. M., Ivanova, K., Amorim, S., Reis, R. L., Pashkuleva, I., Mendoza, E., Pfeifer, A., Heinze, T. and Tzanov, T. Acta Biomater. 2016, 33, 203-212.

29. Awsiuk, K., Budkowski, A., Psarouli, A., Petrou, P., Bernasik, A., Kakabakos, S., Rysz, J. and Raptis, I. Colloid Surface B 2013, 110, 217-224.

30. Hildebrandt, P. and Stockburger, M. J. Phys. Chem-US 1984, 88, 5935-5944.

31. Mogensen, K. B. and Kneipp, K. J. Phys. Chem. C 2014, 118, 28075-28083.

32. Jiang, L.-P., Xu, S., Zhu, J.-M., Zhang, J.-R., Zhu, J.-J. and Chen, H.-Y. Inorg. Chem. 2004, 43, 5877-5883.

33. Cubillana-Aguilera, L. M., Franco-Romano, M., Gil, M. L. A., Naranjo-Rodríguez, I., Hidalgo-Hidalgo de Cisneros, J. L. and Palacios-Santander, J. M. Ultrason. Sonochem. 2011, 18, 789-794.

34. Luque, R., Ojeda, M., Garcia, A., Lastres, C., Campos, R., Pineda, A., Romero, A. A. and Yepez, A. RSC Adv. 2013, 3, 7119-7123.

35. Arancon, R. A. D., Lin, S. H. T., Chen, G., Lin, C. S. K., Lai, J., Xu, G. and Luque, R. RSC Adv. 2014, 4, 17114-17119. 
36. Fernandes, M. M., Ivanova, K., Francesko, A., Mendoza, E. and Tzanov, T. Process Biochem. 2016, DOI: 10.1016/j.procbio.2016.09.011

37. Jacobsen, S. M., Stickler, D. J., Mobley, H. L. T. and Shirtliff, M. E. Clin. Microbiol. Rev. 2008, 21, 26-59.

38. Donlan, R. M. Emerg. Infect. Dis. 2001, 7, 277-281.

39. Seil, J. T. and Webster, T. J. Int. J. Nanomed. 2012, 7, 2767-2781. 